\title{
Transcriptional changes in Toxoplasma gondii in response to treatment with monensin
}

\author{
Bintao Zhai ${ }^{1,2}$, Jun-Jun He${ }^{2}$, Hany M. Elsheikha ${ }^{3}$, Jie-Xi Li ${ }^{2}$ Xing-Quan Zhu ${ }^{2,4^{*}}$ and Xiaoye Yang ${ }^{1 *}$
}

Background: Infection with the apicomplexan protozoan parasite T. gondii can cause severe and potentially fatal cerebral and ocular disease, especially in immunocompromised individuals. The anticoccidial ionophore drug monensin has been shown to have anti-Toxoplasma gondii properties. However, the comprehensive molecular mechanisms that underlie the effect of monensin on T. gondii are still largely unknown. We hypothesized that analysis of T. gondii transcriptional changes induced by monensin treatment can reveal new aspects of the mechanism of action of monensin against T. gondii.

Methods: Porcine kidney (PK)-15 cells were infected with tachyzoites of T. gondii RH strain. Three hours post-infection, PK-15 cells were treated with $0.1 \mu \mathrm{M}$ monensin, while control cells were treated with medium only. PK-15 cells containing intracellular tachyzoites were harvested at 6 and $24 \mathrm{~h}$ post-treatment, and the transcriptomic profiles of $T$. gondii-infected PK-15 cells were examined using high-throughput RNA sequencing (RNA-seq). Quantitative real-time PCR was used to verify the expression of 15 differentially expressed genes (DEGs) identified by RNA-seq analysis.

Results: A total of 4868 downregulated genes and three upregulated genes were identified in monensin-treated T. gondii, indicating that most of T. gondii genes were suppressed by monensin. Kyoto Encyclopedia of Genes and Genomes (KEGG) pathway enrichment analysis of T. gondii DEGs showed that T. gondii metabolic and cellular pathways were significantly downregulated. Spliceosome, ribosome, and protein processing in endoplasmic reticulum were the top three most significantly enriched pathways out of the 30 highly enriched pathways detected in T. gondii. This result suggests that monensin, via down-regulation of protein biosynthesis in T. gondii, can limit the parasite growth and proliferation.

Conclusions: Our findings provide a comprehensive insight into T. gondii genes and pathways with altered expression following monensin treatment. These data can be further explored to achieve better understanding of the specific mechanism of action of monensin against $T$. gondii.

Keywords: Toxoplasma gondii, Monensin, PK-15 cells, RNA-sequencing

\section{Background}

Toxoplasma gondii is one of the most successful opportunistic pathogens and has a wide range of intermediate

\footnotetext{
*Correspondence: xingquanzhu1@hotmail.com; xiaoyeyang@imau.edu.cn ${ }^{1}$ College of Veterinary Medicine, Inner Mongolia Agricultural University, Hohhot 010018, Inner Mongolia Autonomous Region, People's Republic of China

2 State Key Laboratory of Veterinary Etiological Biology, Key Laboratory of Veterinary Parasitology of Gansu Province, Lanzhou Veterinary Research Institute, Chinese Academy of Agricultural Sciences, Lanzhou 730046, Gansu, People's Republic of China

Full list of author information is available at the end of the article
}

hosts [1, 2]. This prolific parasite is estimated to cause latent infection in a third of the global human population [3]. While T. gondii is largely benign in immunocompetent individuals, infection with this parasite can cause severe inflammation of the retina, and in severely immunosuppressed patients, latent tissue cysts can reactivate in the brain causing life-threatening toxoplasmic encephalitis [4]. Toxoplasma gondii is also responsible for significant economic losses attributed to abortions of pregnant sheep following primary infection especially during early and mid-pregnancy [5].

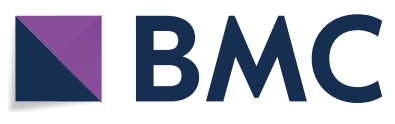

(c) The Author(s) 2020. This article is licensed under a Creative Commons Attribution 4.0 International License, which permits use, sharing, adaptation, distribution and reproduction in any medium or format, as long as you give appropriate credit to the original author(s) and the source, provide a link to the Creative Commons licence, and indicate if changes were made. The images or other third party material in this article are included in the article's Creative Commons licence, unless indicated otherwise in a credit line to the material. If material is not included in the article's Creative Commons licence and your intended use is not permitted by statutory regulation or exceeds the permitted use, you will need to obtain permission directly from the copyright holder. To view a copy of this licence, visit http://creativeco mmons.org/licenses/by/4.0/. The Creative Commons Public Domain Dedication waiver (http://creativecommons.org/publicdomain/ zero/1.0/) applies to the data made available in this article, unless otherwise stated in a credit line to the data. 
In veterinary medicine, control of ovine toxoplasmosis relies on the use of decoquinate [6]. Also, monensin [7] and the folate inhibitor drugs, sulphamezathine and pyrimethamine [8], have been evaluated against $T$. gondii infection in pregnant sheep. There is a vaccine (Toxovax ${ }^{\circledR}$, MSD Animal health) licensed for the prevention of abortion in sheep [9], although this vaccine suffers from a number of shortcomings [10]. Regarding humans, the first-line therapy for $T$. gondii infection is a combination of pyrimethamine and sulfadiazine. However, this regimen has some limitations because these drugs must be taken for a long duration, often cause side effects, and are incapable of eliminating the latent infection [11]. These drawbacks pose a major obstacle in conventional chemotherapy of toxoplasmosis in humans. To this end, efforts have been made to identify new and more effective medicines $[12,13]$ and to understand the mechanism of action [14] and perturbation associated with the currently used drugs [15].

One of the drugs that received more attention in recent years is monensin, which is an ionophore antibiotic used to treat coccidiosis in poultry and dairy animals. Monensin has shown antiparasitic activity against $T$. gondii in vitro $[16,17]$ and in sheep [7]. Through induction of oxidative stress, monensin disrupts the mitochondrial function, and induces an arrest of the cell cycle and autophagy-like cell death in T. gondii [14]. Given the promising anti- $T$. gondii activity of monensin, further understanding of its mechanism of action could reveal new targets for drug development against $T$. gondii. The transcriptomic profile of $T$. gondii-infected porcine kidney (PK-15) cells has been reported [18]. However, comprehensive understanding of how monensin treatment alters the transcriptome of $T$. gondii remains unknown.

In the present study, we profiled global gene expression in T. gondii following treatment of T. gondii-infected PK-15 cells with monensin using high-throughput RNA-sequencing (RNA-seq) analysis. Our data showed that monensin can cause genome-wide transcriptional changes in T. gondii.

\section{Methods}

\section{Toxoplasma gondii culture}

Tachyzoites of $T$. gondii RH strain were cultured and maintained in porcine (Sus scrofa) kidney (PK-15) cell monolayers. PK-15 cells were obtained from the American Tissue Culture Collection (ATCC ${ }^{\circledR}$ CCL-33 ${ }^{\mathrm{TM}}$; Maryland, USA) and cultured in Dulbecco's Modified Eagle's Medium (DMEM, HyClone, Shanghai, China) supplemented with $10 \%$ fetal bovine serum (Gibco, Maryland, USA) at $37{ }^{\circ} \mathrm{C}$ in $5 \% \mathrm{CO}_{2}$. Tachyzoites were harvested when $80 \%$ of the infected PK-15 cells had lysed. The infected cells and egressed tachyzoites were passed through a 22-gauge needle 20 times to rupture any remaining PK-15 cells. The supernatant was removed by centrifugation at $350 \times g$ for $10 \mathrm{~min}$ at $4{ }^{\circ} \mathrm{C}$, and the tachyzoites were resuspended in $3 \mathrm{ml}$ DMEM. The final purified tachyzoites were counted using a hemocytometer.

\section{Monensin treatment}

PK-15 cells were infected with tachyzoites at a multiplicity of infection of 3 ( 3 tachyzoites: 1 PK-15 cell). Three hours post-infection, 12 T25 tissue culture flasks were randomly divided into four groups (3 flasks/group). The two treatment groups included M6 (T. gondii-infected cells at $6 \mathrm{~h}$ post-monensin treatment) and M24 (T. gondii-infected cells at $24 \mathrm{~h}$ post-monensin treatment). The two control groups (C6 and C24) were infected and untreated cells. The M6 and M24 groups were treated with monensin solution (Alfa Aesar, Ward Hill, USA) at a final concentration of $0.1 \mu \mathrm{M}$, while the control groups were treated with fresh medium without monensin. Each group included three biological replicates. The treated and control (untreated) cells were harvested at 6 and 24 $\mathrm{h}$ post-treatment and stored at $-80^{\circ} \mathrm{C}$, until used for RNA extraction and RNA-seq.

\section{RNA extraction and RNA-seq analysis}

Total RNA was individually extracted from each sample using TRIzol (Invitrogen China Ltd, Beijing, China) according to the manufacturer's instructions. All extracted RNAs were treated with RNase-Free DNase (Ambion, Shanghai, China) to remove any residual genomic DNA. The integrity and quantity of all RNA samples were examined using the Agilent 2100 Bioanalyzer (Agilent Technologies, Santa Clara, CA, USA) and a NanoDrop ${ }^{\mathrm{TM}}$ spectrophotometer (Thermo Scientific, Wilmington, DE, USA), respectively. Five micrograms of total RNA were used for the construction of the transcriptome libraries and 100-bp paired-end strand-specific RNA-sequencing was performed on the BGISEQ-500 Platform as per the manufacturer's instructions.

\section{Sequence filtering, read mapping and analysis of differentially expressed genes (DEGs)}

The raw sequencing data were processed using the FASTX tool (http://hannonlab.cshl.edu/fastx_toolkit/) to remove adaptor sequences, low-quality reads (quality value $<20$ ), reads containing $>5 \% \mathrm{~N}$ rate and joint sequences before downstream analyses. StringTie [19] was used to reconstruct the transcripts guided by the genomic annotation information. Novel transcripts were identified using Cuffcompare (a tool of Cufflinks) [20]. The coding ability of new transcripts was predicted using Coding Potential Calculator [21]. The highquality clean reads were then mapped to the reference 
genomes of pig (Sus scrofa) (ftp://ftp.ncbi.nlm.nih.gov/ genomes/Sus_scrofa/) and T. gondii (ftp://ftp.ncbi.nlm. nih.gov/genomes/refseq/protozoa/Toxoplasma_gondi i/latest_assembly_versions/GCF_000006565.2_TGA4) using HISAT and Bowtie 2 tools [22]. The gene expression level was calculated for each sample using the RSEM (RNA-seq by expectation-maximization) program [23] and the FPKM (fragments per kilobase of exon per million mapped fragments) method. DEseq2 software was used to identify the differentially expressed genes (DEGs). Gene expression with log2 fold change $\geq 1$ or $\leq-1$, and adjusted $P$-value $<0.01$ was considered as differentially expressed. Universal Protein Resource (UniProt) (https ://www.uniprot.org/), Kyoto Encyclopedia of Genes and Genomes (KEGG) Orthology Based Annotation System 3.0 (KOBAS) (http://kobas.cbi.pku.edu.cn/index.php) and Gene Ontology (GO, http://geneontology.org/) were used for gene/protein functional annotation, pathway annotation and gene enrichment analyses, respectively. The GO enrichment analysis results were categorized according to the biological process (BP), cellular component (CC) and molecular function (MF). The RNA-seq, reads alignment and DEG identification were carried out at BGI-Shenzhen, China.

\section{Verification of RNA-seq results by qPCR}

Quantitative real-time PCR (qPCR) was used to verify the RNA-seq results. The expression levels for 15 DEGs were determined by qPCR using the same RNA samples that were used for the sequencing. The RNA samples were reverse-transcribed to single strand cDNA using the PrimeScript $^{\mathrm{TM}}$ RT reagent Kit (TaKaRa, Dalian, China). Fifteen genes (nine host cell genes and six T. gondii genes) were randomly selected for $\mathrm{qPCR}$ verification and $\beta$-actin was used as the reference gene. All qPCR reactions were performed on the BIO-CFX96 system (Bio-Rad, California, USA) using SYBR Green GoTaq ${ }^{\circledR}$ qPCR Master Mix (Promega, Beijing, China) following the manufacturer's instructions. The primers used for qPCR are listed in the Additional file 1: Table S1. The selected genes were analyzed in triplicate. The qPCR cycling conditions included $95^{\circ} \mathrm{C}$ for $2 \mathrm{~min}$ followed by 40 cycles of $95^{\circ} \mathrm{C}$ for $10 \mathrm{~s}, 58$ ${ }^{\circ} \mathrm{C}$ for $15 \mathrm{~s}, 72{ }^{\circ} \mathrm{C}$ for $40 \mathrm{~s}$, and the temperatures of the melting curve analysis ranged from 72 to $95{ }^{\circ} \mathrm{C}$. The $2^{-}$ $\triangle \Delta \mathrm{Cq}$ method was used to calculate the relative expression of each gene.

\section{Results}

We analyzed the global gene expression of $T$. gondii infecting PK-15 cells in the absence or presence of 0.1 $\mu \mathrm{M}$ monensin treatment using an Illumina platform. The obtained sequences were aligned against pig and T. gondii genome sequences. More than $11.01 \mathrm{~Gb}$ of clean bases/

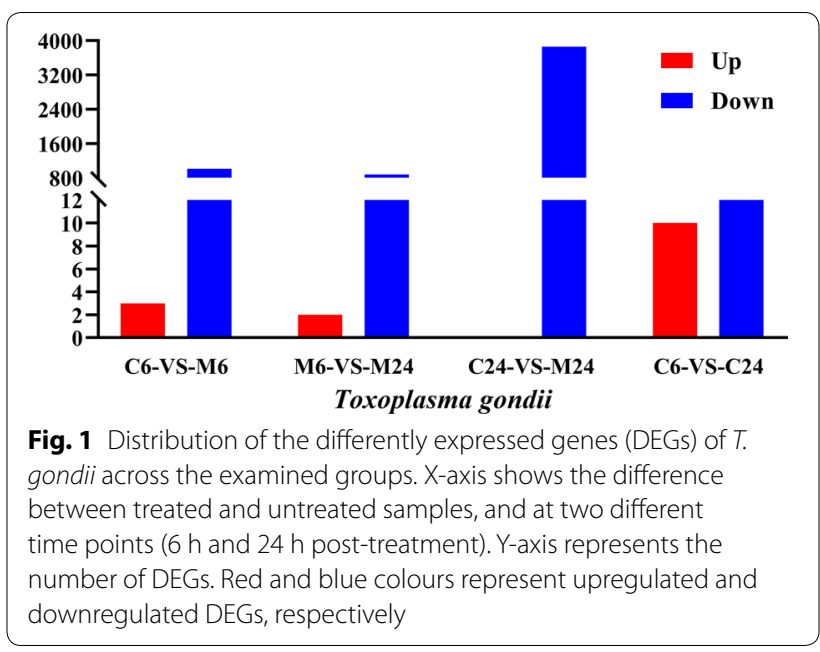

reads were obtained from each treated and untreated sample (Additional file 2: Table S2).

\section{Differentially expressed genes (DEGs)}

Three upregulated and 1012 downregulated T. gondii genes were detected at $6 \mathrm{~h}$ post-treatment, while 3856 downregulated T. gondii genes were found at $24 \mathrm{~h}$ posttreatment (Fig. 1). Interestingly, 990 downregulated $T$. gondii DEGs were shared between monensin-treated samples at 6 and $24 \mathrm{~h}$ (Fig. 2). These 990 downregulated

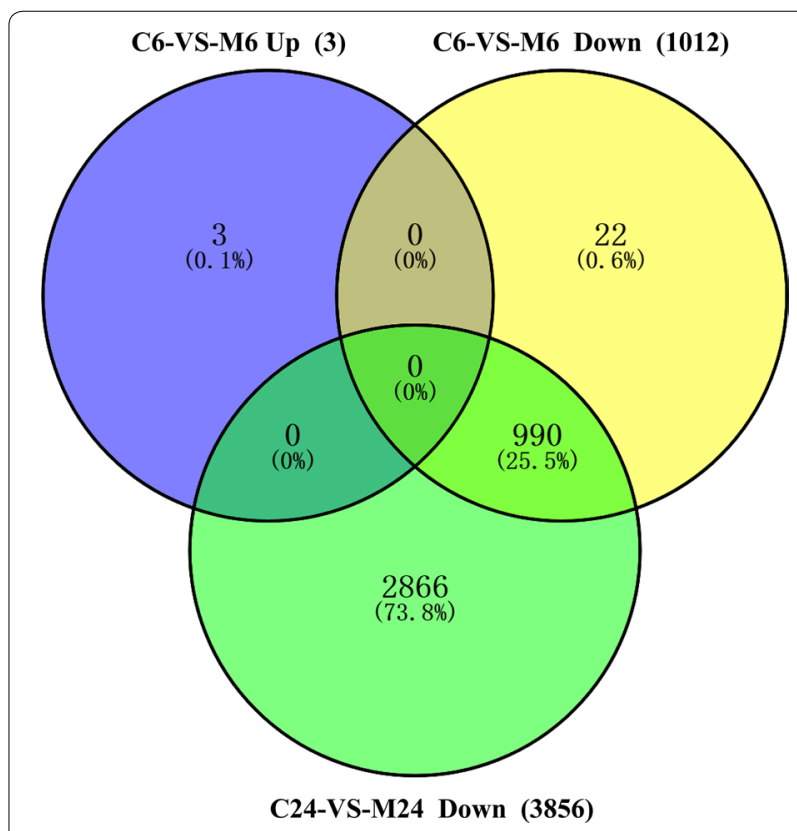

Fig. 2 Venn diagram showing the overlap of the number of up and downregulated genes of T. gondii in C6 vs M6 group (6 h), and C24 vs M24 group (24 h) 
genes accounted for $97.8 \%$ of the downregulated genes at $6 \mathrm{~h}$ and $25.7 \%$ of the downregulated genes at $24 \mathrm{~h}$ posttreatment. The expression of 15 genes obtained through RNA-seq were confirmed by qPCR and the validation results are shown in Fig. 3.

\section{Gene Ontology (GO) analysis of the DEGs}

A total of $44 \mathrm{GO}$ terms, including 17 biological process (BP) terms, 15 cellular component (CC) terms and 12 molecular function (MF) terms were significantly enriched for 4871 T. gondii DEGs (Fig. 4). Among the BP category at 6 and $24 \mathrm{~h}$, the top two enriched GO terms were metabolic process and cellular process. In the CC category at $6 \mathrm{~h}$, membrane and cell were the top two GO terms (Fig. 4a), while membrane and membrane part were the top two GO terms at $24 \mathrm{~h}$ (Fig. $4 \mathrm{~b}$ ). In the MF category at 6 and $24 \mathrm{~h}$, the top two GO terms were catalytic activity and binding.

\section{KEGG pathway analysis}

We also mapped the DEGs to six different KEGG subsystems, including metabolism, genetic information processing, environmental information processing, cellular processes, organismal systems and human diseases (Fig. 5). KEGG pathway analysis also showed that most of T. gondii DEGs were enriched in infectious diseases, signal transduction and translation. The 30 most significantly enriched pathways are shown in Fig. 6; spliceosome, ribosome, and protein processing in endoplasmic reticulum are the top three significantly enriched pathways in T. gondii (Additional file 3: Figure S1, Additional file 4: Figure S2, Additional file 5: Figure S3).

\section{Transcription factors (TFs) of DEGs}

TFs are key regulators of gene expression [24]. They bind to specific DNA sequences and activate or repress gene expression by DNA-binding domains (DBDs) [25]. Based on their DBDs, TFs can be classified into different families [26]. In our study, differentially expressed TFs were classified into 25 families (Fig. 7), and homeobox and zf-C2H2 were the two most significantly enriched TFs in T. gondii.

\section{Protein-protein interaction (PPI) of DEGs}

Using the String database prediction, PPI networks of T. gondii with a combined score $>980$ at $6 \mathrm{~h}$ post-monensin treatment are shown in Fig. 8. TGME49_002580 (XM_018779214.1), which encodes ATPases associated with diverse cellular activities (AAA proteins), was the most enriched upregulated gene in T. gondii. Four proteins, TGME49_238180 (XM_018780522.1, K03037), TGME49_292220 (XM_002368522.2, K03033), TGME49_250830 (XM_018780938.1, K03031) and TGME49_227960 (XM_002366378.2, K03036), formed a separate mutual network. TGME49_238180, TGME49_292220 and TGME49_227960 regulate TGME49_250830, while TGME49_292220 regulates TGME49_250830, TGME49_227960 and TGME49_238180. These proteins (TGME49_292220 (K03033, Rpn3), TGME49_238180 (K030037, Rpn7), TGME49_227960 (K03036, Rpn6), and TGME49_250830 (K03031, Rpn12)) are the components of the $19 \mathrm{~S}$ regulatory particle in the proteasome pathway (map03050, Additional file 6: Figure S4). The protein TGME49_289830 (XM_002368367.1, K03246) regulates two proteins, TGME49_294670 (XM_002370195.2, K03248) and TGME49_317720 (XM_018782917.1, K03251); where they all belong to the family of translation initiation factors (eIF3) of the RNA transport pathway. Additional file 7: Figure S5 shows T. gondii PPIs at $24 \mathrm{~h}$ where TGME49_210790 (XM_002371193.2), TGME49_266460 (XM_002368694.2), TGME49_297140 (XM_018782303.1), TGME49_275750 (XM_002371561.2) and TGME49_305010 (XM_002370254.1) are some of the proteins that warrant further studies.

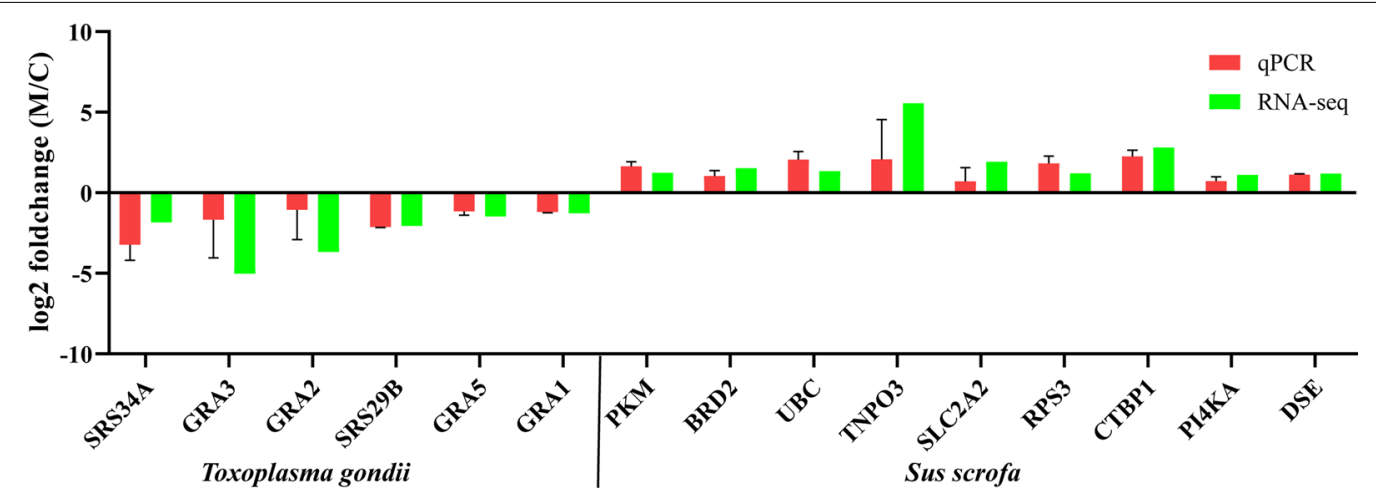

Fig. 3 Verification of the RNA-seq data by using qPCR. Bars represent the mean fold changes of the expression of six T. gondii genes and nine porcine genes 
a

Level2 GO terms of all

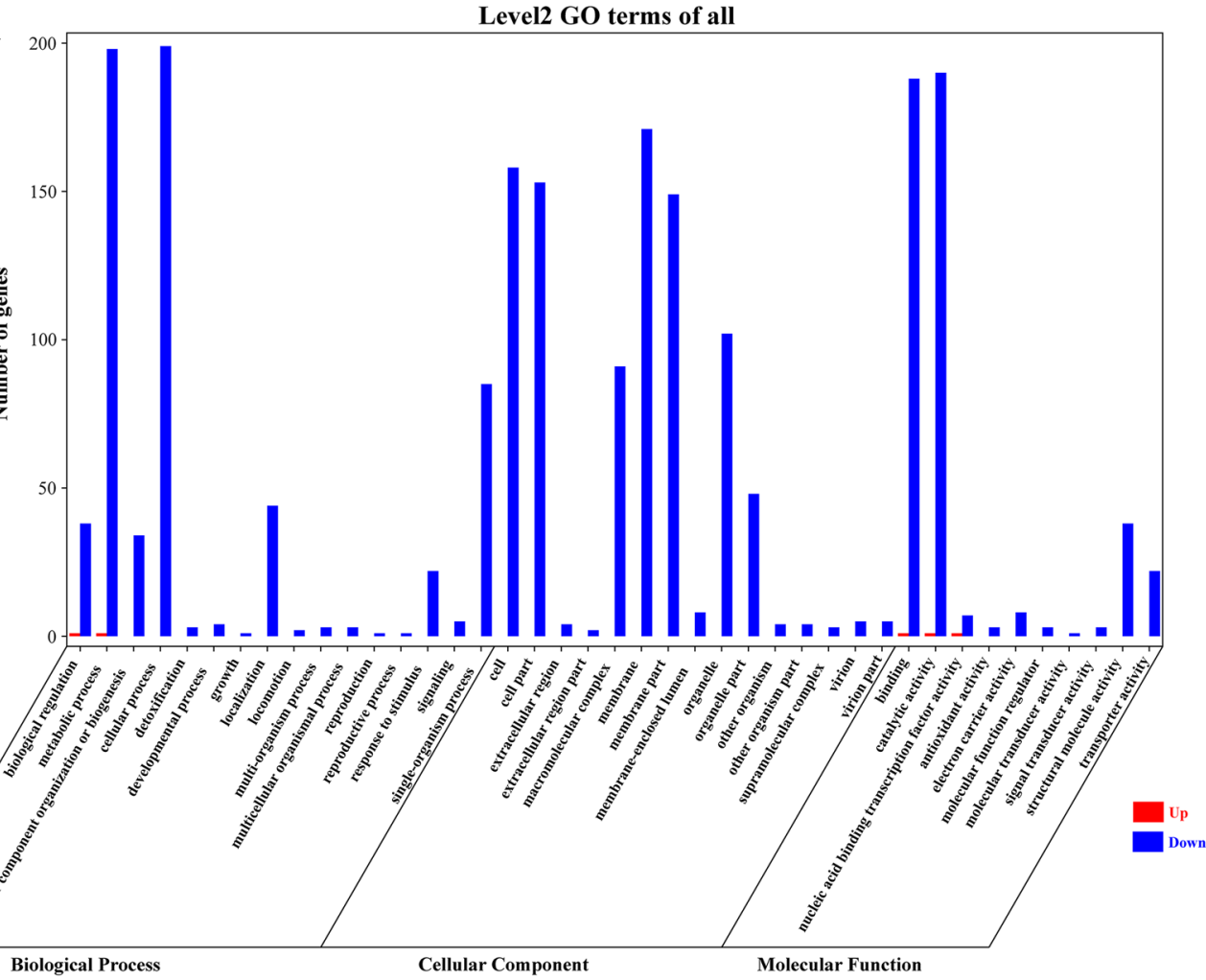

b

Level2 GO terms of all

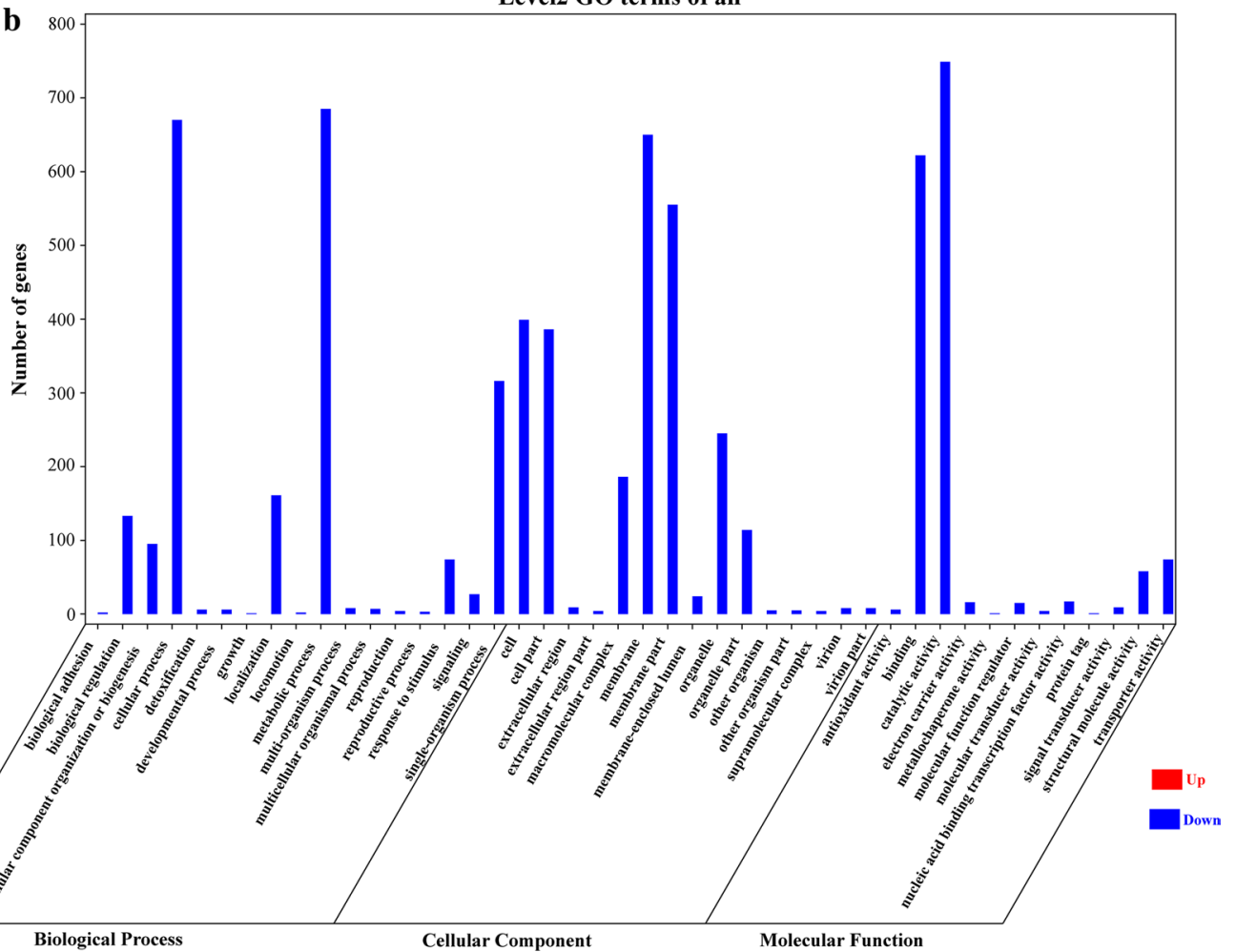

Fig. $4 \mathrm{GO}$ enrichment analysis of the differentially expressed genes (DEGs) of T. gondii. The bar graphs show the number of T. gondii DEGs enriched in $\mathrm{GO}$ terms belonging to the three GO categories, biological process, cellular component and molecular function, at $6 \mathrm{~h}$ (a) and $24 \mathrm{~h}$ (b). X-axis represents the $\mathrm{GO}$ terms and $\mathrm{Y}$-axis represents the number of upregulated (Up) and downregulated (Down) genes in different $\mathrm{GO}$ terms 


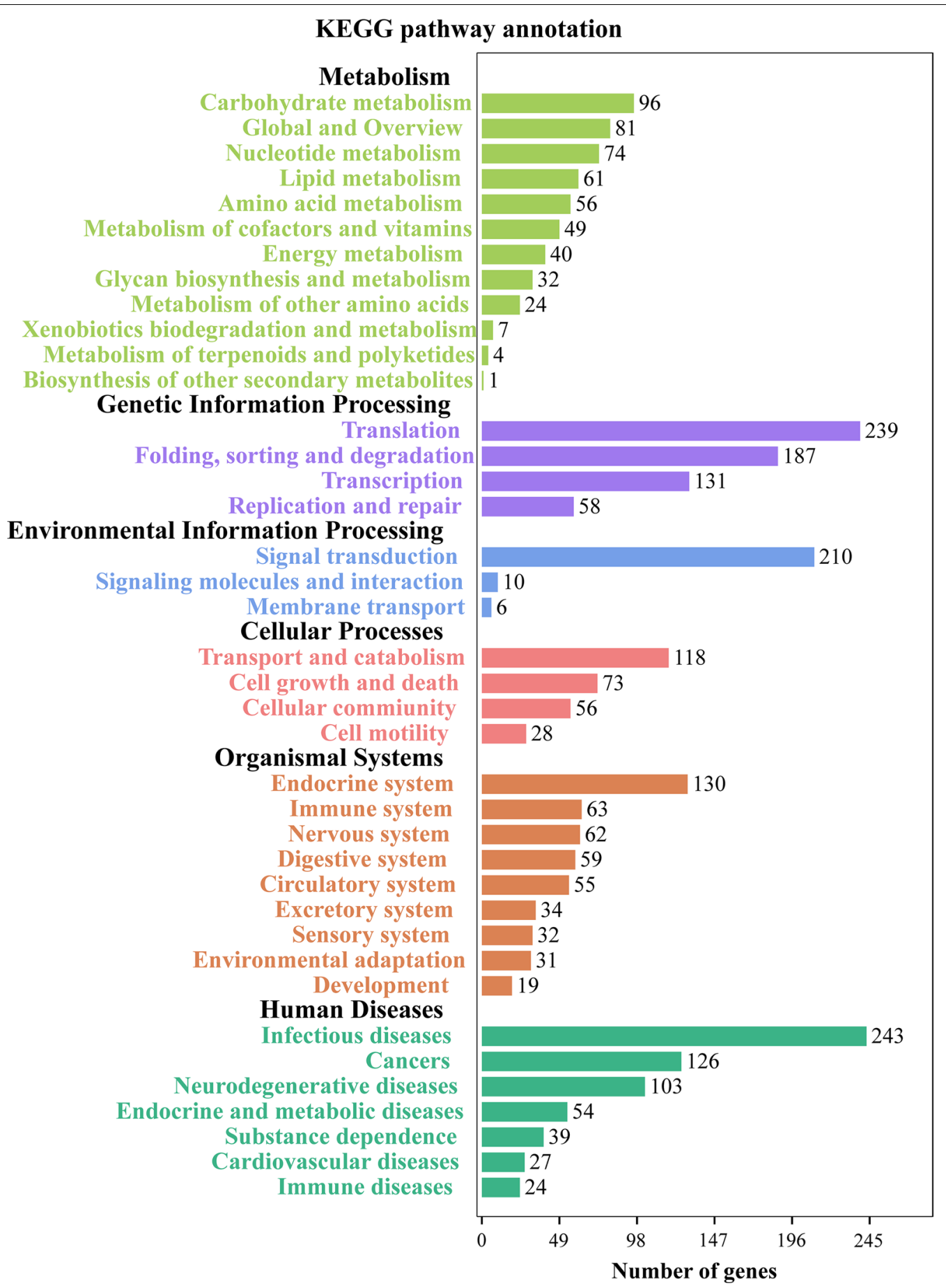

Fig. 5 KEGG annotation of the DEGs in the transcriptome of T. gondii. X-axis label represents the number of DEGs in the corresponding KEGG pathways in each KEGG subsystem. Y-axis label represents main clusters of the KEGG pathways

\section{Discussion}

The search for new anti-Toxoplasma gondii drugs has been active for several decades [12, 13], but only a few drugs are currently approved for use in humans $[1,27]$. Although sulfa drugs can be effectively used for the prevention and control of T. gondii infection in humans and animals, their side effects should not be ignored [28]. Compared to the mainstream anti-Toxoplasma drugs (sulfa and ethylamines, trimethoprim combined with sulfamethoxazole), monensin seems to be less cytotoxic [29, 


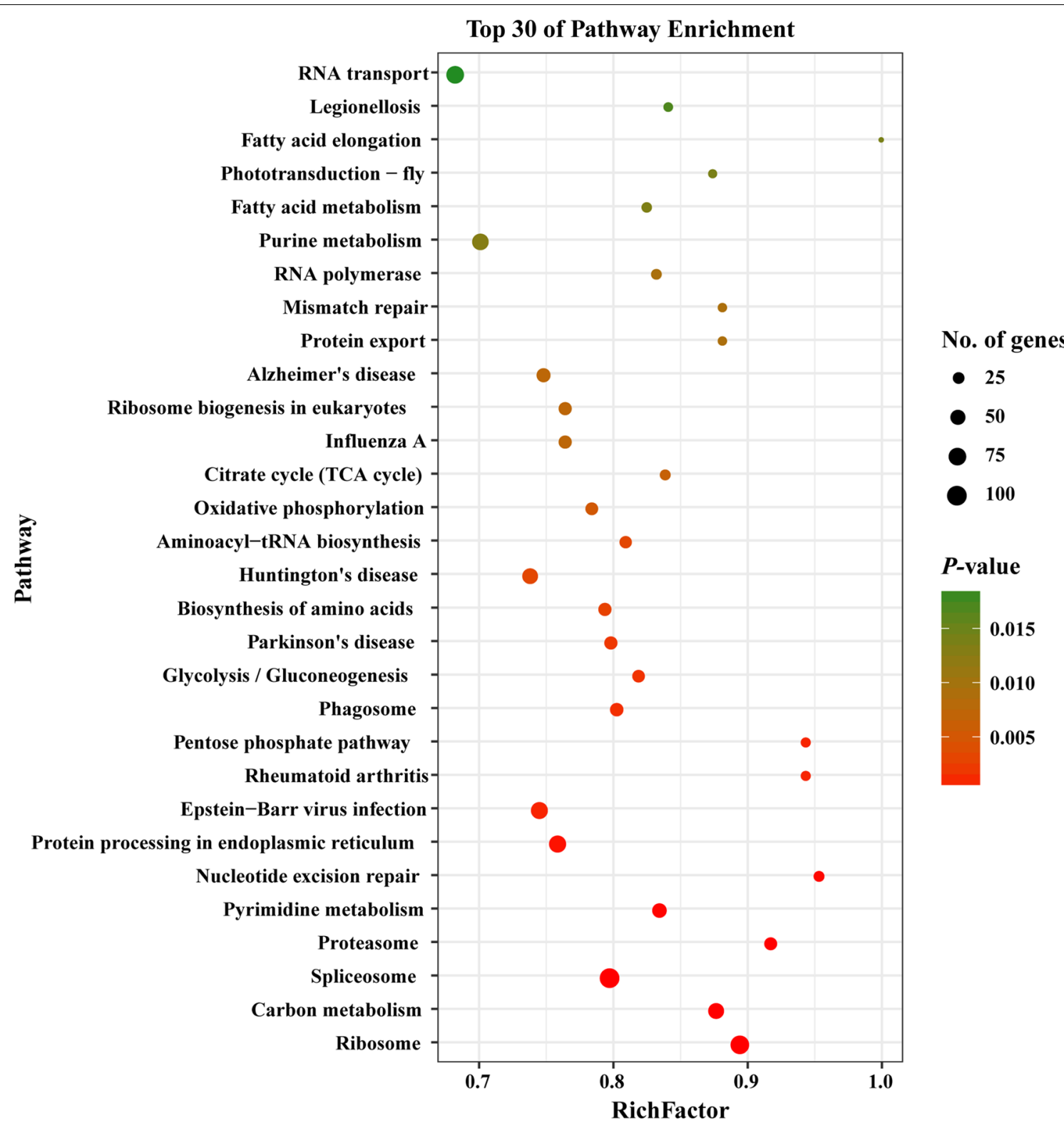

Fig. 6 Scatterplot of the top 30 most enriched KEGG pathways of T. gondii. Y-axis label represents the distinct KEGG pathways, and X-axis label represents the Rich Factor. Rich Factor refers to the ratio of DEGs annotated in the pathway to total number of genes annotated in the pathway. The greater the Rich Factor, the greater the degree of pathway enrichment. Dot size represents the number of DEGs (bigger dots denote large DEG number and vice versa). The colours of the dots represent the $P$-values of enrichment. Red colour indicates high enrichment, while green colour indicates low enrichment

30]. The anticoccidial drug monensin has been shown to inhibit the viability and even damage the bradyzoite stage of T. gondii [29] and to prevent the shedding of oocysts from cats [31]. Monensin can also induce cell cycle arrest and autophagy, leading to death of $T$. gondii tachyzoites $[32,33]$, probably mediated by an oxidative stress-related mechanism [14]. Despite this body of literature describing the mechanisms that mediate the inhibitory effects of monensin against different life-cycle forms of $T$. gondii, the comprehensive mechanisms responsible for killing of T. gondii by monensin remains incompletely defined.

In this study, we used RNA-seq technology to identify the global transcriptomic changes in $T$. gondii caused by monensin treatment. We found 4868 downregulated genes and three upregulated genes in T. gondii following monensin treatment. The significant number of 


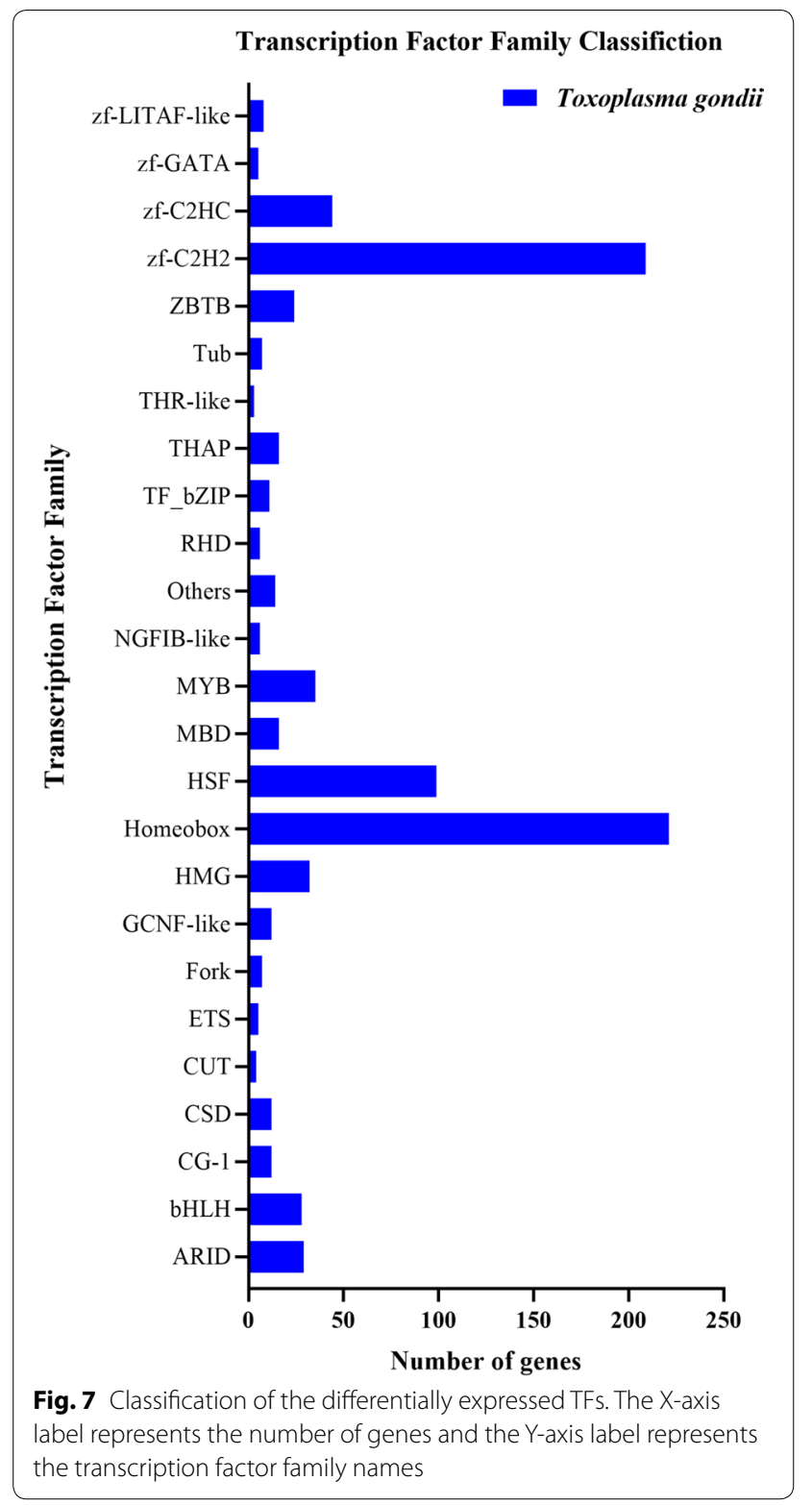

downregulated genes shows the overwhelming impact of monensin treatment on $T$. gondii, especially at $24 \mathrm{~h}$ posttreatment. We also performed GO enrichment analysis to analyze the significantly altered biological processes in T. gondii caused by monensin treatment. The two most significantly enriched BP GO terms at 6 and $24 \mathrm{~h}$ were metabolic process and cellular process. In the MF category, the top two GO terms were catalytic activity and binding at 6 and $24 \mathrm{~h}$. For the CC category, membrane and membrane parts were the two most enriched GO terms at both 6 and $24 \mathrm{~h}$ (Fig. 4); these included membrane components that contribute to material transportation, membrane integration, environmental resistance and various biological functions that are essential for cell survival. These findings indicate that anti- $T$. gondii effects of monensin could be mediated by impairment of most of $T$. gondii biological processes and membrane components.

KEGG pathway analysis showed that spliceosome, ribosome and protein processing in the endoplasmic reticulum were the top three of the 30 most significantly enriched pathways in T. gondii (Fig. 6). Protein processing in the endoplasmic reticulum is a pathway that influences protein folding in the endoplasmic reticulum [34]. Proteolytic cleavage of effectors in the endoplasmic reticulum pathway is essential for the survival of T. gondii [35]. Additional file 5: Figure S3 shows that most genes involved in protein processing in the endoplasmic reticulum pathway are downregulated. Thus, we infer that monensin could suppress protein processing in the endoplasmic reticulum pathway in $T$. gondii, which would contribute to its anti-T. gondii activity.

The spliceosomes are RNA-protein complexes responsible for removal of introns (non-coding segments) from pre-messenger RNAs to form mature mRNAs in a process known as splicing [36]. Spliceosome components have been identified in T. gondii [37]. Our analysis showed that all DEGs involved in the spliceosome pathway are downregulated by monensin (Additional file 3: Figure S1). Ribosome biogenesis is closely related to multiple cellular signaling pathways and any defects in ribosome production can cause many diseases, and even death [38]. The ribosome profiling at the level of transcription and translation of T. gondii has been reported [39]. However, how the ribosome of T. gondii is altered by monensin remains unknown. Our analysis showed that DEGs involved in ribosome biogenesis are significantly downregulated by monensin (Additional file 4: Figure S2). These findings indicate that monensin can also interfere with genes involved in mRNA translation and ribosome biogenesis, which can restrict the growth of $T$. gondii.

The biogenesis of the spliceosome and ribosome are regulated by transcription factors (TFs). We found that homeobox and $\mathrm{zf}-\mathrm{C} 2 \mathrm{H} 2$ were the two most significantly enriched TFs (Fig. 7). The homeobox TF regulates the expression of genes associated with various developmental processes in animals, fungi and plants [40]. The zf$\mathrm{C} 2 \mathrm{H} 2 \mathrm{TF}$ family contains a small protein structural motif, the zinc finger (zf), which coordinates one or more zinc ions $\left(\mathrm{Zn}_{2}^{+}\right)$[41]. TFs containing zinc fingers have been implicated in a variety of biological processes in T. gondii $[42,43]$. For example, depletion of TgZNF2 in $T$. gondii caused an arrest of the parasite growth at the G1 phase of the cell cycle and accumulation of poly(A) RNA in their nucleus [43]. Thus, monensin-induced downregulation 


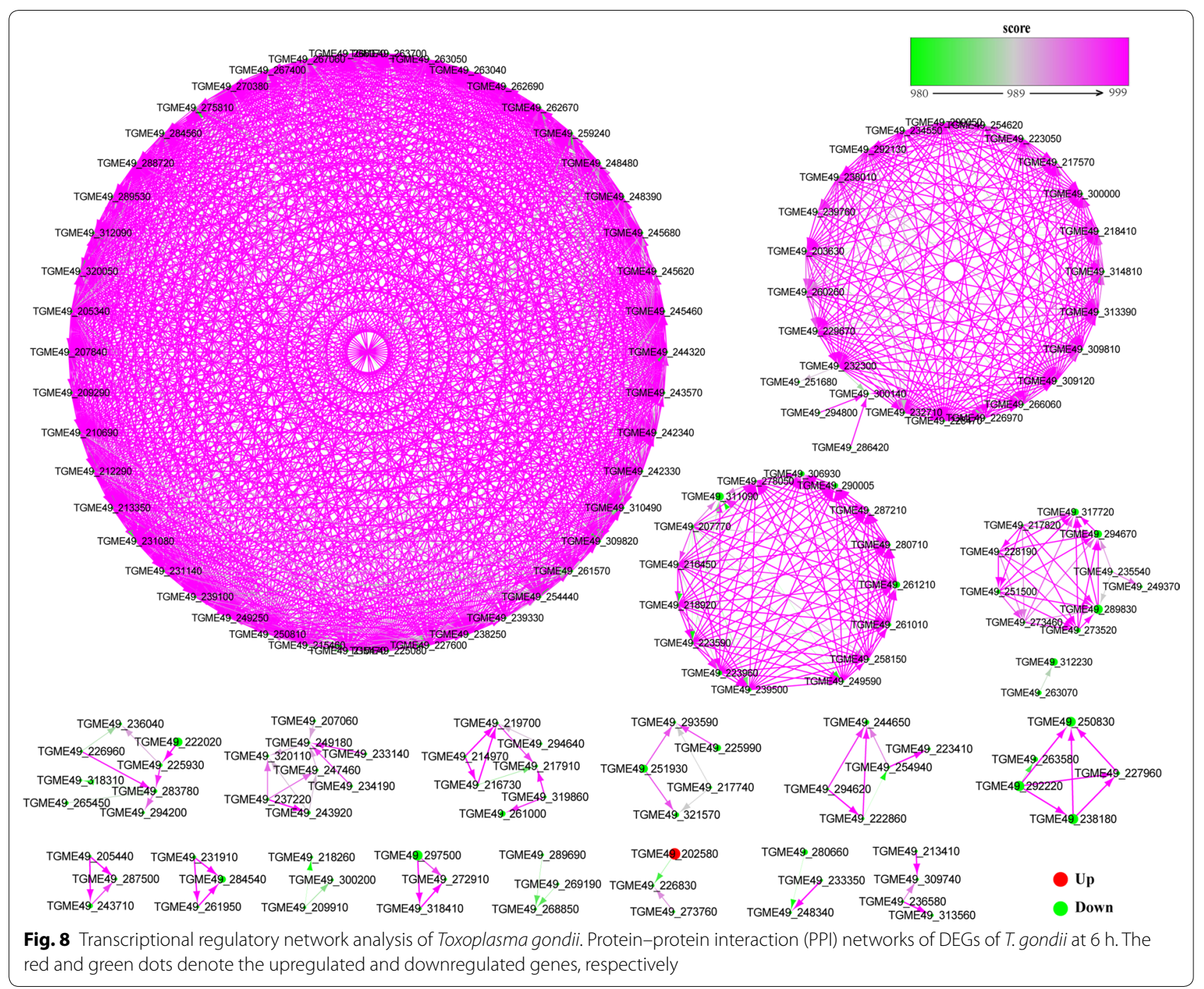

of these two TFs, homeobox and $\mathrm{zf}-\mathrm{C} 2 \mathrm{H} 2$, may disrupt the growth and development of $T$. gondii, further elucidating more aspects of monensin mode of action against T. gondii.

The PPI analysis revealed several proteins that were downregulated by monensin, including TGME49_210790, TGME49_305010, TGME49_266460 and TGME49_002580. TGME49_002580 is ATPase, AAA family protein, which plays critical roles in various cellular processes [44]. TGME49_210790 (XM_002371193.2) encodes a putative dihydroorotate dehydrogenase (DHODH), which mediates the fourth step of de novo pyrimidine biosynthesis [45]. In $T$. gondii, disruption of de novo pyrimidine synthesis results in uracil auxotrophy, virulence attenuation and inability to establish latent infection [46]. Inhibition of the activity of $T$. gondii dihydroorotate dehydrogenase (TgDHODH) can potentiate the growth inhibiting potential of 1-hydroxyquinolones in T. gondii [45]. TGME49_305010 (XM_002370254.1) is putatively encoded as pre-mRNA branch site protein p14, which is associated with U2 small nuclear ribonucleoprotein particles (snRNPs) and participates in the spliceosome (map03040) pathway. TGME49_266460 (XM_002368694.2) encodes a small ubiquitin-like family modifier (SUMO) belonging to the Ubl family, while only one gene is encoded by SUMO in lower eukaryotes, including T. gondii [47]. A previous study of $T$. gondii SUMO proteomics revealed over 100 sumoylated proteins involved in translation, metabolism, posttranslational modification, and protein degradation [48]. Altering these proteins in T. gondii may be lethal, which would then contribute to the anti- $T$. gondii activity of monensin. 


\section{Conclusions}

This study examined the transcriptomic landscape of T. gondii infecting PK-15 cells treated with monensin and identified monensin-induced DEGs in T. gondii. Our genome-wide transcriptional analysis revealed that 4868 T. gondii genes were downregulated in treated cell cultures, suggesting that monensin can suppress the expression of the majority of T. gondii genes. Also, monensin treatment appears to adversely influence various crucial metabolic and cellular processes of $T$. gondii, such as spliceosome, ribosome and protein processing in the endoplasmic reticulum. Additionally, monensin induced downregulation of two transcription factors, homeobox and zf-C2H2, in T. gondii. Further analysis of the identified transcriptional changes can provide useful information for better understanding of the mechanism of action of monensin against $T$. gondii.

\section{Supplementary information}

Supplementary information accompanies this paper at https://doi. org/10.1186/s13071-020-3970-1.

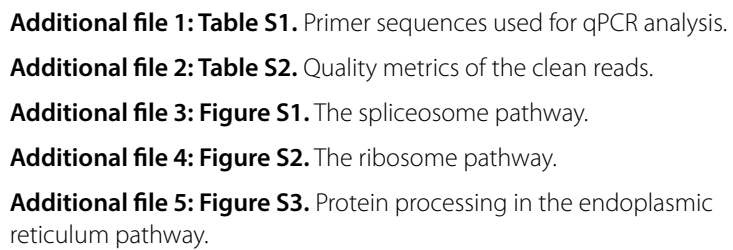

Additional file 6: Figure S4. Proteasome pathway (map03050). The proteasome is a protein-destroying apparatus involved in many essential cellular functions. The green box Rpn3 represents TGME49_292220 (K03033), Rpn7 represents TGME49_238180 (K030037), Rpn6 represents TGME49_227960 (K03036), and Rpn12 represents TGME49_250830 (K03031).

Additional file 7: Figure S5. Toxoplasma gondii PPIs at $24 \mathrm{~h}$ post-treatment.

\begin{abstract}
Abbreviations
qPCR: Quantitative real-time PCR; DEGs: Differentially expressed genes; TE: Toxoplasmic encephalitis; CNS: Central nervous system; PK-15: Porcine kidney-15; ATCC: American Tissue Culture Collection; DMEM: Dulbecco's Modified Eagle's Medium; MOI: Multiplicity of infection; KEGG: Kyoto Encyclopedia of Genes and Genomes; GO: Gene Ontology; BP: Biological process; CC: Cellular component; MF: Molecular function; RNA-seq: RNA-sequencing; FPKM: Fragments per kilobase of exon per million mapped fragments; TFs: Transcription factors; DBD: DNA-binding domain; PPI: Protein-protein interactions; zf: Zinc finger; C2H2: Cys2His2-like fold group.
\end{abstract}

\section{Acknowledgements}

We thank BGI-Shenzhen for technical assistance.

\section{Authors' contributions}

$X Y Y, J J H, H M E$ and XQZ conceived and designed the study, and critically revised the manuscript. BTZ performed the experiment, analyzed the transcriptomic data and drafted the manuscript. JJH and JXL helped in the study implementation and data analysis. All authors read and approved the final manuscript.

\section{Funding}

Project support was kindly provided by the International Science and Technology Cooperation Project of Gansu Provincial Key Research and Development Program (Grant No. 17JR7WA031), the Elite Program of Chinese Academy of Agricultural Sciences, and the Agricultural Science and Technology Innovation Program (ASTIP) (Grant No. CAAS-ASTIP-2016-LVRI-03).

\section{Availability of data and materials}

The RNA-seq data obtained in this study were deposited in the National Center for Biotechnology Information (NCBI) Sequence Read Archive (SRA) database (https://www.ncbi.nlm.nih.gov/sra) under accession number SUB6209220.

\section{Ethics approval and consent to participate}

Not applicable.

\section{Consent for publication}

Not applicable.

\section{Competing interests}

The authors declare that they have no competing interests.

\section{Author details}

${ }^{1}$ College of Veterinary Medicine, Inner Mongolia Agricultural University, Hohhot 010018, Inner Mongolia Autonomous Region, People's Republic of China. ${ }^{2}$ State Key Laboratory of Veterinary Etiological Biology, Key Laboratory of Veterinary Parasitology of Gansu Province, Lanzhou Veterinary Research Institute, Chinese Academy of Agricultural Sciences, Lanzhou 730046, Gansu, People's Republic of China. ${ }^{3}$ Faculty of Medicine and Health Sciences, School of Veterinary Medicine and Science, University of Nottingham, Sutton Bonington Campus, Loughborough LE12 5RD, UK. ${ }^{4}$ Jiangsu Co-innovation Center for the Prevention and Control of Important Animal Infectious Diseases and Zoonoses, Yangzhou University College of Veterinary Medicine, Yangzhou 225009, Jiangsu, People's Republic of China.

Received: 22 September 2019 Accepted: 13 February 2020

Published online: 18 February 2020

\section{References}

1. Dunay IR, Gajurel K, Dhakal R, Liesenfeld O, Montoya JG. Treatment of toxoplasmosis: historical perspective, animal models, and current clinical practice. Clin Microbiol Rev. 2018;31:e00057-17.

2. Wang ZD, Wang SC, Liu HH, Ma HY, Li ZY, Wei F, et al. Prevalence and burden of Toxoplasma gondii infection in HIV-infected people: a systematic review and meta-analysis. Lancet HIV. 2017;4:e177-88.

3. Flegr J, Prandota J, Sovickova M, Israili ZH. Toxoplasmosis-a global threat. Correlation of latent toxoplasmosis with specific disease burden in a set of 88 countries. PLOS ONE. 2014;9:e90203.

4. Blader IJ, Coleman BI, Chen CT, Gubbels MJ. Lytic cycle of Toxoplasma gondii: 15 years later. Annu Rev Microbiol. 2015;69:463-85.

5. Webster JP. Review of "Toxoplasmosis of Animals and Humans (Second Edition)" by JP Dubey. Parasites Vectors. 2010;3:112.

6. Buxton D, Brebner J, Wright S, Maley SW, Thomson KM, Millard K. Decoquinate and the control of experimental ovine toxoplasmosis. Vet Rec. 1996;138:434-6.

7. Buxton D, Blewett DA, Trees AJ, McColgan C, Finlayson J. Further studies in the use of monensin in the control of experimental ovine toxoplasmosis. J Comp Pathol. 1988;98:225-36.

8. Buxton D, Thomson KM, Maley S. Treatment of ovine toxoplasmosis with a combination of sulphamezathine and pyrimethamine. Vet Rec. 1993;132:409-11.

9. Buxton D, Thomson KM, Maley S, Wright S, Bos HJ. Experimental challenge of sheep 18 months after vaccination with a live (S48) Toxoplasma gondii vaccine. Vet Rec. 1993;133:310-2.

10. Wang JL, Zhang NZ, Li TT, He JJ, Elsheikha HM, Zhu XQ. Advances in the development of anti-Toxoplasma gondii vaccines: challenges, opportunities, and perspectives. Trends Parasitol. 2019;35:239-53. 
11. Chen QW, Dong K, Qin HX, Yang YK, He JL, Li J, et al. The direct and indirect inhibition effects of resveratrol against Toxoplasma gondii tachyzoites in vitro. Antimicrob Agents Chemother. 2019;63:e01233-18.

12. Montazeri M, Sharif M, Sarvi S, Mehrzadi S, Ahmadpour E, Daryani A. A systematic review of in vitro and in vivo activities of anti-Toxoplasma drugs and compounds (2006-2016). Front Microbiol. 2017;8:25.

13. Wei HX, Wei SS, Lindsay DS, Peng HJ. A systematic review and metaanalysis of the efficacy of anti-Toxoplasma gondii medicines in humans. PLoS ONE. 2015;10:e0138204.

14. Charvat RA, Arrizabalaga G. Oxidative stress generated during monensin treatment contributes to altered Toxoplasma gondii mitochondrial function. Sci Rep. 2016;6:22997.

15. Zhou CX, Gan Y, Elsheikha HM, Chen XQ, Cong H, Liu Q, et al. Sulfadiazine sodium ameliorates the metabolomic perturbation in mice infected with Toxoplasma gondii. Antimicrob Agents Chemother. 2019;63:e00312-9.

16. Lavine MD, Arrizabalaga G. Analysis of monensin sensitivity in Toxoplasma gondii reveals autophagy as a mechanism for drug induced death. PLoS ONE. 2012;7:e42107.

17. Thabet A, Schmidt J, Baumann S, Honscha W, Von Bergen M, Daugschies $A$, et al. Resistance towards monensin is proposed to be acquired in a Toxoplasma gondii model by reduced invasion and egress activities, in addition to increased intracellular replication. Parasitology. 2018;145:313-25.

18. Zhou CX, Elsheikha HM, Zhou DH, Liu Q, Zhu XQ, Suo X. Dual identification and analysis of differentially expressed transcripts of porcine PK-15 cells and Toxoplasma gondii during in vitro infection. Front Microbiol. 2016;7:721.

19. Pertea M, Pertea GM, Antonescu CM, Chang TC, Mendell JT, Salzberg SL. StringTie enables improved reconstruction of a transcriptome from RNAseq reads. Nat Biotechnol. 2015;33:290-5.

20. Trapnell C, Roberts A, Goff L, Pertea G, Kim D, Kelley DR, et al. Differential gene and transcript expression analysis of RNA-seq experiments with TopHat and Cufflinks. Nat Protoc. 2012;7:562-78.

21. Kong L, Zhang Y, Ye ZQ, Liu XQ, Zhao SQ, Wei L, et al. CPC: assess the protein-coding potential of transcripts using sequence features and support vector machine. Nucleic Acids Res. 2007;35:W345-9.

22. Langmead B, Salzberg SL. Fast gapped-read alignment with Bowtie 2. Nat Methods. 2012;9:357-9.

23. Li B, Dewey CN. RSEM: accurate transcript quantification from RNA-Seq data with or without a reference genome. BMC Bioinform. 2011;12:323.

24. Lambert M, Jambon S, Depauw S, David-Cordonnier MH. Targeting transcription factors for cancer treatment. Molecules. 2018;23:E1479.

25. Elshan NGRD, Rettig MB, Jung ME. Molecules targeting the androgen receptor $(A R)$ signaling axis beyond the AR-Ligand binding domain. Med Res Rev. 2019;39:910-60.

26. Charoensawan V, Wilson D, Teichmann SA. Genomic repertoires of DNAbinding transcription factors across the tree of life. Nucleic Acids Res. 2010:38:7364-77.

27. Neville AJ, Zach SJ, Wang X, Larson JJ, Judge AK, Davis LA, et al. Clinically available medicines demonstrating anti-Toxoplasma activity. Antimicrob Agents Chemother. 2015;59:7161-9.

28. Deng Y, Wu T, Zhai SQ, Li CH. Recent progress on anti-Toxoplasma drugs discovery: design, synthesis and screening. Eur J Med Chem. 2019;183:111711.

29. Couzinet S, Dubremetz JF, Buzoni-Gatel D, Jeminet G, Prensier G. In vitro activity of the polyether ionophorous antibiotic monensin against the cyst form of Toxoplasma gondii. Parasitology. 2000;121:359-65.

30. Lawrence K. Use of monensin sodium against Toxoplasma. Vet Rec. 1988;123:323.
31. Frenkel JK, Smith DD. Inhibitory effects of monensin on shedding of Toxoplasma oocysts by cats. J Parasitol. 1982;68:851-5.

32. Varberg JM, LaFavers KA, Arrizabalaga G, Sullivan WJ. Characterization of Plasmodium Atg3-Atg8 interaction inhibitors identifies novel alternative mechanisms of action in Toxoplasma gondii. Antimicrob Agents Chemother. 2018;62:e01489-17.

33. Lavine MD, Arrizabalaga G. The antibiotic monensin causes cell cycle disruption of Toxoplasma gondii mediated through the DNA repair enzyme TgMSH-1. Antimicrob Agents Chemother. 2011;55:745-55.

34. Beauvais G, Bode NM, Watson JL, Wen H, Glenn KA, Kawano H, et al. Disruption of protein processing in the endoplasmic reticulum of DYT1 knock-in mice implicates novel pathways in dystonia pathogenesis. J Neurosci. 2016:36:10245-56.

35. Coffey MJ, Jennison C, Tonkin CJ, Boddey JA. Role of the ER and golgi in protein export by apicomplexa. Curr Opin Cell Biol. 2016;41:18-24.

36. Hoskins AA, Friedman LJ, Gallagher SS, Crawford DJ, Anderson EG, Wombacher R, et al. Ordered and dynamic assembly of single spliceosomes. Science. 2011:331:1289-95.

37. Gubbels MJ, Wieffer M, Striepen B. Fluorescent protein tagging in Toxoplasma gondii: identification of a novel inner membrane complex component conserved among Apicomplexa. Mol Biochem Parasitol. 2004;137:99-110.

38. Pelava A, Schneider C, Watkins NJ. The importance of ribosome production, and the 5S RNP-MDM2 pathway, in health and disease. Biochem Soc Trans. 2016:44:1086-90.

39. Holmes MJ, Shah P, Wek RC, Sullivan WJ. Simultaneous ribosome profiling of human host cells infected with Toxoplasma gondii. mSphere. 2019;4:e00292-19.

40. Leite DJ, Baudouin-Gonzalez L, Iwasaki-Yokozawa S, Lozano-Fernandez J, Turetzek N, Akiyama-Oda Y, et al. Homeobox gene duplication and divergence in arachnids. Mol Biol Evol. 2018;35:2240-53.

41. Ryu Y, Kang JA, Kim D, Kim SR, Kim S, Park SJ, et al. Programed assembly of nucleoprotein nanoparticles using DNA and zinc fingers for targeted protein delivery. Small. 2018;14:e1802618.

42. Dubey JP. The history of Toxoplasma gondii - the first 100 years. J Eukaryot Microbiol. 2008:55:467-75.

43. Gissot M, Hovasse A, Chaloin L, Schaeffer-Reiss C, Van Dorsselaer A, Tomavo S. An evolutionary conserved zinc finger protein is involved in Toxoplasma gondii mRNA nuclear export. Cell Microbiol. 2017; 19:e12644.

44. Suvorova ES, Radke JB, Ting LM, Vinayak S, Alvarez CA, Kratzer S, et al. A nucleolar AAA-NTPase is required for parasite division. Mol Microbiol. 2013;90:338-55.

45. Hegewald J, Gross U, Bohne W. Identification of dihydroorotate dehydrogenase as a relevant drug target for 1-hydroxyquinolones in Toxoplasma gondii. Mol Biochem Parasitol. 2013;190:6-15.

46. Fox BA, Bzik DJ. Nonreplicating, cyst-defective type II Toxoplasma gondii vaccine strains stimulate protective immunity against acute and chronic infection. Infect Immun. 2015;83:2148-55.

47. Crater AK, Roscoe S, Fahim A, Ananvoranich S. Toxoplasma ubiquitin-like protease 1, a key enzyme in sumoylation and desumoylation pathways, is under the control of non-coding RNAs. Int J Parasitol. 2018;48:867-80.

48. Braun L, Cannella D, Pinheiro AM, Kieffer S, Belrhali H, Garin J, et al. The small ubiquitin-like modifier (SUMO)-conjugating system of Toxoplasma gondii. Int J Parasitol. 2009;39:81-90.

\section{Publisher's Note}

Springer Nature remains neutral with regard to jurisdictional claims in published maps and institutional affiliations. 